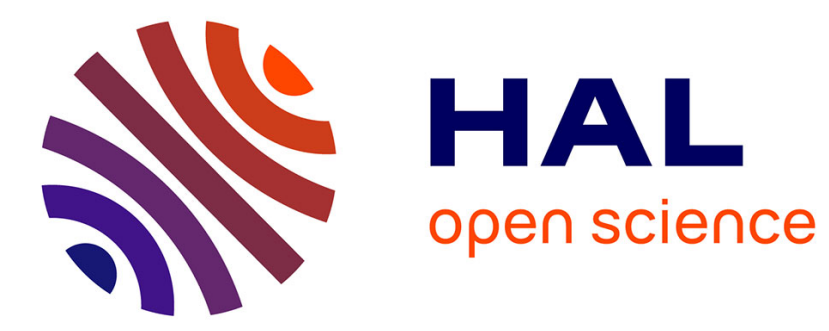

\title{
Integration of UAS in Terminal Control Area
}

Cyril Allignol, Nicolas Barnier, Nicolas Durand, Guido Manfredi, Éric Blond

\section{To cite this version:}

Cyril Allignol, Nicolas Barnier, Nicolas Durand, Guido Manfredi, Éric Blond. Integration of UAS in Terminal Control Area. DASC 2016 IEEE/AIAA 35th Digital Avionics Systems Conference, Sep 2016, Sacramento, United States. 10.1109/DASC.2016.7778052 . hal-01418171

\section{HAL Id: hal-01418171 \\ https://hal-enac.archives-ouvertes.fr/hal-01418171}

Submitted on 16 Dec 2016

HAL is a multi-disciplinary open access archive for the deposit and dissemination of scientific research documents, whether they are published or not. The documents may come from teaching and research institutions in France or abroad, or from public or private research centers.
L'archive ouverte pluridisciplinaire HAL, est destinée au dépôt et à la diffusion de documents scientifiques de niveau recherche, publiés ou non, émanant des établissements d'enseignement et de recherche français ou étrangers, des laboratoires publics ou privés. 


\section{Integration of UAS in Terminal Control Area}

\author{
Cyril Allignol, Nicolas Barnier, Nicolas Durand, Guido Manfredi \\ ENAC \\ 7, av. Édouard Belin \\ 31055 Toulouse, France \\ firstname.surname@enac.fr
}

\author{
Éric Blond \\ DSNA/DTI \\ 1, av. du Dr Maurice Grynfogel \\ 31035 Toulouse, France \\ firstname.surname@aviation-civile.gouv.fr
}

\begin{abstract}
In this article, we test a horizontal detect and avoid algorithm for UASs flying in Terminal Control Areas. We have used recorded commercial traffic trajectories and randomly built thousands of conflict scenarios with UASs to check the ability of such an algorithm to ensure the separation with commercial aviation. We consider two different types of UASs, flying at $80 \mathrm{kn}$ or $160 \mathrm{kn}$, with six different missions: flying straight or turning and leveled, climbing or descending. We only focus on horizontal maneuvers at constant speed in order to not interfere with the TCASs of aircraft, nor rely on most UASs poor ability to change speed. The article investigates the influence of the various parameters on the separation achieved and the amount of maneuvers required, especially the strategy used to select the best maneuver among the allowed headings. The analysis of our results shows that, amid two basic and "extreme" strategies that favor either minimal heading changes or the robustness of the maneuvers, the combination of both, switching from the first one to the second whenever the distance between the UAS and aircraft falls under a given threshold, gives the best results with very few remaining airproxes, while keeping low the amount and amplitude of maneuvers.

Index Terms-UAS, self-separation, conflict resolution, geometrical algorithm.
\end{abstract}

\section{INTRODUCTION}

In the last years, the demand to operate civilian UASs for very diverse missions (ranging from fire detection and river bed surveillance to small items delivery and archaeological exploration...) has rocketed [1]. Most of these UASs operate within the lower airspace (under FL180) and their trajectories may interfere with commercial aviation traffic in Terminal Control Areas (TCA). Developing new algorithms to help separating UASs from the rest of the traffic is becoming critical for safety reasons. In this context, several approaches could be investigated.

First, separation can be entirely managed by Air Navigation Service Providers. Experiments [2] have shown that Air Traffic Controllers resolution process is hindered by UASs mixed with conventional traffic because they have unusual performance specifications and interact with different time responses.

Otherwise, separation could be delegated to both commercial aircraft and UASs which could autonomously maneuver to resolve potential conflicts. However, complex processes of coordination should be considered in such a context to keep Air Traffic Controllers aware of the resolution process and able to interfere in it.

Finally, conflict resolution could be taken care of by UASs only such that they do not disrupt the commercial traffic. This seems a more realistic approach, provided that the positions and speeds of surrounding aircraft are available (through ADSB for example) and that the performances of UASs and the resolution anticipation are sufficient to solve all traffic situations. In this article, we investigate the latter by adapting a self-separation algorithm used in robotics to our context and experiment with various parameters and strategies on real traffic samples.

1) Detect \& Avoid: This algorithm was designed by van den Berg et al. [3] and tested with different speed constraint hypotheses by Durand et al. [4] in the context of autonomous air conflict resolution. Compared to these first experiments, we here tailor the geometrical approach of van den Berg further to model the performances of UASs and consider specific fallback strategies to handle cases for which the first approach fails to maintain separation.

More specifically, adapting the algorithm to integrate UASs in commercial traffic leads to the following major differences from the preliminary work presented in [4]:

- The UAS is here supposed to avoid other aircraft, which do not avoid the UAS. This means that the whole avoidance maneuver is endorsed by the UAS.

- UASs used in a civilian context generally fly with low speeds compared to commercial aircraft. The ratio we used in this article can go from 1.5 to 5 . We focus on the lower airspace where the aircraft speed is theoretically limited at $250 \mathrm{kn}$, but recorded data show that in practice some aircraft fly much faster (up to $400 \mathrm{kn}$ ). In this study, we consider two types of UASs: Fast UASs flying at $160 \mathrm{kn}$ and Slow UASs flying at $80 \mathrm{kn}$.

- Moreover, most civilian UASs have very poor speed up performances compared to conventional aircraft. We will therefore only consider maneuvers at constant speed for UASs, as this degree of freedom would have almost no effect on the resolution process with realistic traffic.

- Commercial aircraft flying in the lower airspace are generally climbing or descending and their speeds are constantly changing, either increasing when climbing, or decreasing when descending and changing direction as well. This factor has a great influence on the detect and avoid strategy in order to ensure that a reasonable distance to the encountered traffic can be maintained. Using real traffic data is therefore essential to validate a resolution algorithm for such evolving and intricate traffic. 
- Air traffic trajectory prediction, which is one of the main component of a conflict solver, is always tainted with uncertainties that must be taken into account to assess the efficiency of an algorithm. We show how our approach can handle uncertainties by providing robust resolution maneuvers.

2) Related Works: When the concept of Free-flight emerged in the 90 s, one of the ideas was then to equip every aircraft with a detect and avoid algorithm able to ensure separation with the rest of the traffic.

The first effective approach used sliding forces to coordinate maneuvers between aircraft [5]. Potential or vortex fields [6] as well as a model based on an analogy with electrical particle repulsion [7] were also used. In 2001, we proposed a token allocation strategy combined with an $A^{*}$ algorithm to solve conflicts with realistic maneuvers [8], [9]. Even if some maneuvers could be simultaneously decided, a complete ranking of aircraft was necessary and finding an optimal ranking has been shown to be problem-dependent [10]. We also tried artificial Neural Networks on the two-aircraft problem [11] but they could not be generalized to handle more aircraft. All these approaches have been tested on en-route traffic, mainly with leveled aircraft.

Geometrical algorithms have also been widely studied in robotics [3], [12]-[14]. The powerful technique developed by Van den Berg et al. [3] can handle thousands of agents in a small space. It was applied to aircraft by Snape et al. [14], but the hypotheses of the algorithm require simultaneous vertical and horizontal speed changes. We also tested them [4] in the horizontal plane with speed constraints and showed that this algorithm is unable to deal with high densities of traffic when the speed norm cannot be changed.

More generally, conflict resolution has been proven to be a highly combinatorial optimization problem [15]. Most centralized approaches that have been proposed to solve conflicts can be broadly divided into two main categories. The first ones [16]-[18] use greedy sequential algorithms to optimize trajectories one by one after ranking the aircraft (ordering aircraft is however very challenging [10]). The others try to find the global optimum without the need to prioritize aircraft. Among this second category, many models define aircraft trajectories through simple analytic expressions that introduce strong limits on the type of situations that can be dealt with, as the ones described in [19]-[24]. In [25], [26], we proposed a model to solve multiple aircraft conflicts based on Metaheuristics (Genetic Algorithm and Tabu Search) using trajectory simulation with uncertainties. However, these works mainly targeted en-route traffic control and used simulated traffic only with the BADA model on real flight plans.

3) Outline: In this article, we come back to a simpler problem in a more realistic environment. We consider UASs flying in the lower airspace (under FL180) and design various conflict scenarios with real recorded commercial aircraft trajectories in TCAs. The aim of the study is to assess the performances of a "detect and avoid" strategy for UASs to maintain a reasonable horizontal separation with commercial traffic. We first consider

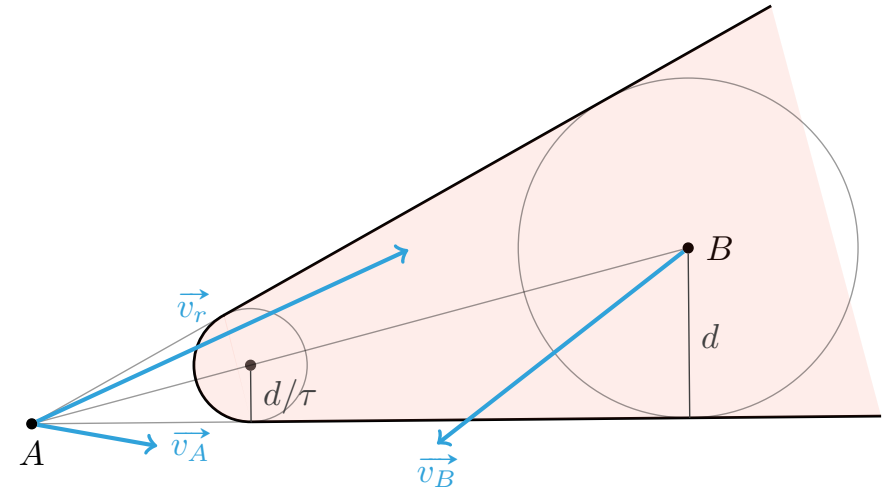

Fig. 1. Conflicting aircraft model: a conflict will occur within time $\tau$ if and only if the relative speed $\overrightarrow{v_{r}}$ lies in the forbidden zone in red.

conflicts involving one UAS and one commercial aircraft only, then we introduce multiple aircraft encountering the UAS.

In section II of this article, we detail the geometrical approach developed by Van den Berg et al. [3] in the case of a single UAS avoiding non cooperative aircraft. We show how the algorithm can be adapted to take into account the constant speed constraint and propose several strategies to choose the maneuver when a future conflict is detected. We also introduce a fallback scheme that reduces the targeted separation distance when no solution is available to maintain separation. Moreover, we describe how our tailored model can be easily extended to take more than two aircraft into account. Finally, we show how some uncertainties on trajectory prediction can be simply taken into account in our model. In section [III-A we describe how we built the conflict scenarios from real traffic data and the hypotheses that were chosen for the UAS. In section III-B, we give some results obtained on the different scenarios and show the influence of the various parameters on the quality of the results in terms of separations achieved and maneuvers imposed on the UAS. The last section draws some conclusions about the results obtained with the simulations and highlights directions for future work.

\section{Detect And Avoid Model}

This section describes the algorithm developed in [3] and its adaptation to the case where only one aircraft (the UAS) maneuvers. We first detail the model with a single aircraft and a minimizing strategy that requires to change the speed of the UAS, then show how the speed of the maneuvering UAS can be constrained to have a constant norm at the cost of greater heading deviations. We also describe a fallback strategy to minimize the separation violation when our model has no solution within the allowed turning range. Our model is then further extended to simultaneously take into account several aircraft while keeping the UAS speed constant.

\section{A. Single Aircraft}

Let $d$ be the standard separation between aircraft and $\tau$ be a look ahead time. In figure 1, let us consider UAS $A$ and 


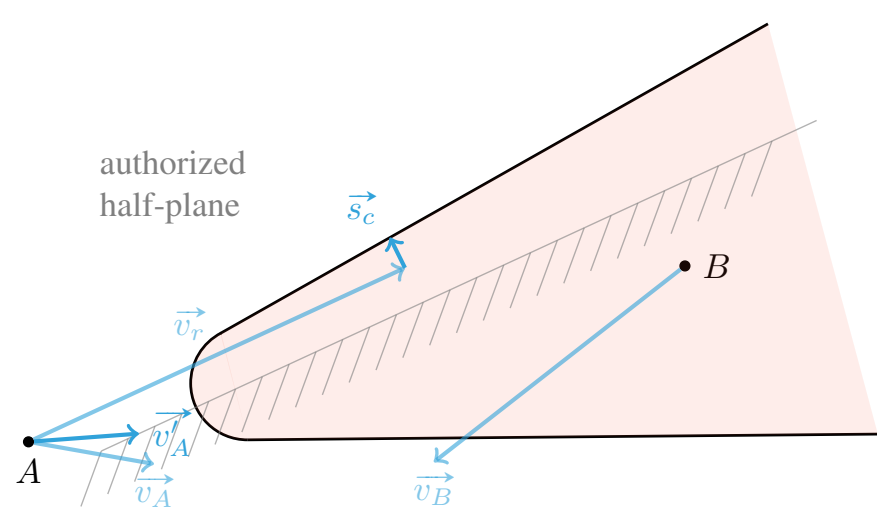

Fig. 2. Conflict resolution model: the necessary speed change to move $\overrightarrow{v_{r}}$ out of the forbidden zone is endorsed by the UAS.

aircraft $B$. We can represent the position of aircraft $B$ in the referential of UAS $A$. If we draw a circle of radius $d$ centered at aircraft $B$, the two lines issued from position $A$, tangent to the circle of radius $d$ form a cone. If the relative speed $\overrightarrow{v_{r}}=\overrightarrow{v_{A}}-\overrightarrow{v_{B}}$ lies in this cone, a conflict will occur in the future. If we draw a circle of size $\frac{d}{\tau}$ tangent to the two previous lines, we obtain a new zone (in light red) bounded by the bold line in figure 1 . It is then straightforward to understand that a conflict will occur within time $\tau$ if and only if $\overrightarrow{v_{r}}$ lies in this zone.

When a potential conflict occurs, i.e. the endpoint of $\overrightarrow{v_{r}}$ lies in the forbidden zone, $\overrightarrow{v_{r}}$ must be changed to escape it. If the speed change is not constrained (by the performances of the aircraft for example), $\overrightarrow{v_{r}}$ can be projected on the nearest bold line (see figure 2) to solve the conflict pairwise between UAS $A$ and aircraft $B$ while minimizing the speed change. In [3], this change is shared in half by both aircraft. Here, we constrain the necessary speed change $\overrightarrow{s_{c}}$ that moves $\overrightarrow{v_{r}}$ out of the forbidden zone to be entirely attributed to UAS $A$.

We can then define a half-plane bounded by the line going through point $A+\overrightarrow{v_{A}}+\overrightarrow{s_{c}}$ parallel to the corresponding edge of the cone on the side determined by $\overrightarrow{s_{c}}$ : if the new speed of the UAS $\overrightarrow{v_{A}^{\prime}}$ is chosen such that its endpoint lies in this halfplane, then the new relative speed $\overrightarrow{v_{r}}$ is out of the forbidden speed zone, as illustrated on figure 2 .

However, this minimizing strategy generally results in changing the norm of the speed of the UAS, which is not realistic considering the average performances of commercial UASs. We show in section II-B how to take this additional constraint into account in our geometrical model.

\section{B. Constant Speed}

We consider here that a UAS can only change its heading and not its speed norm. In reality, a UAS can change its speed norm, but the change rate is very limited and almost negligible with respect to the speed of surrounding aircraft (typically airliners).

To ensure that the norm of the speed of the UAS remains constant throughout the conflict resolution process, we must

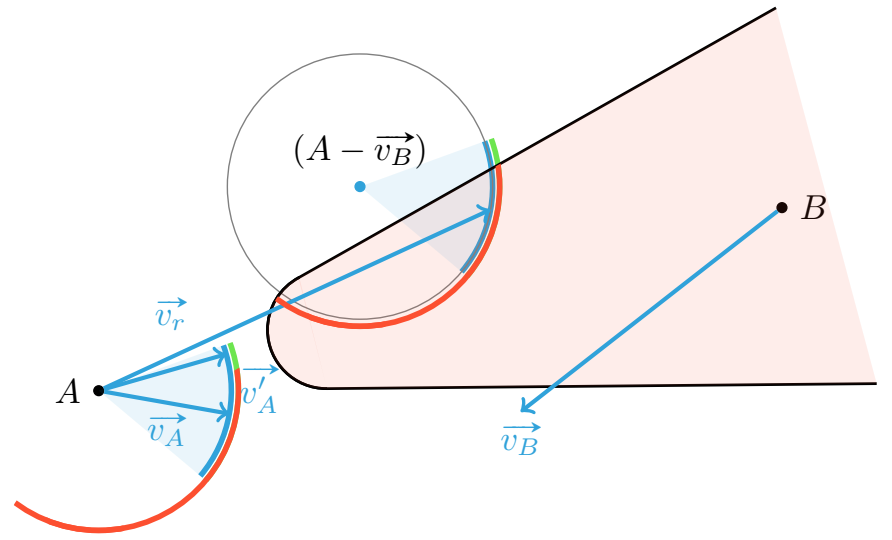

Fig. 3. Model with constant speed norm for the UAS: the end of $\overrightarrow{v_{r}^{\prime}}$ must lie on the circle but outside of the forbidden zone (in light red) and in the allowed turn angle range of $30^{\circ}$ around $\overrightarrow{v_{A}}$, which leaves the tiny green safe zone as possible new heading for the UAS.

have:

$$
\left\|\overrightarrow{v_{A}^{\prime}}\right\|=\left\|\overrightarrow{v_{A}}\right\|
$$

as $\overrightarrow{v_{r}^{\prime}}=\overrightarrow{v_{A}^{\prime}}-\overrightarrow{v_{B}}$, we also have:

$$
\left(x_{v_{r}^{\prime}}+x_{v_{B}}\right)^{2}+\left(y_{v_{r}^{\prime}}+y_{v_{B}}\right)^{2}=\left\|\overrightarrow{v_{A}}\right\|^{2}
$$

which means that the possible endpoints of $\overrightarrow{v_{r}^{\prime}}$ belongs to a circle of radius $\left\|\overrightarrow{v_{A}}\right\|$ centered at $A-\overrightarrow{v_{B}}$ as shown on figure 3 But $\overrightarrow{v_{r}^{\prime}}$ must also lies outside the forbidden zone defined in the previous section, which removes all angle ranges of the circle included in it (in red on the figure). The remaining angles must be further filtered by intersecting the allowed turn angle range $\theta$ corresponding to the performance of the UAS, i.e. an arc of $\pm \theta$ around the current speed shown in blue on figure 3 (where $\theta=30^{\circ}$ ).

The conflict-free heading change thus ranges over an arc of a circle (pictured in green on figure 3) which is the difference of the allowed (in blue) and forbidden (in red) arcs. Note that the resulting allowed headings may comprise two disjoint angle ranges, which is generally the case for facing aircraft for example (as shown on figure 4) and corresponds to the combinatorial decision to turn right or left to solve the future conflict.

\section{Heading Change Strategies}

As mentioned in the previous section, the conflict-free solution set is generally not a singleton, so a decision must be taken to choose the "best" angle within possibly discontinuous angle ranges.

1) Closest: The new speed for the UAS can be aggressively chosen with the available angle that minimizes the heading change, which will result in optimizing the "maneuvers quantity" (a measure defined in section III-B). This strategy will be referred to as Closest in the remaining sections and is pictured in figure 5 


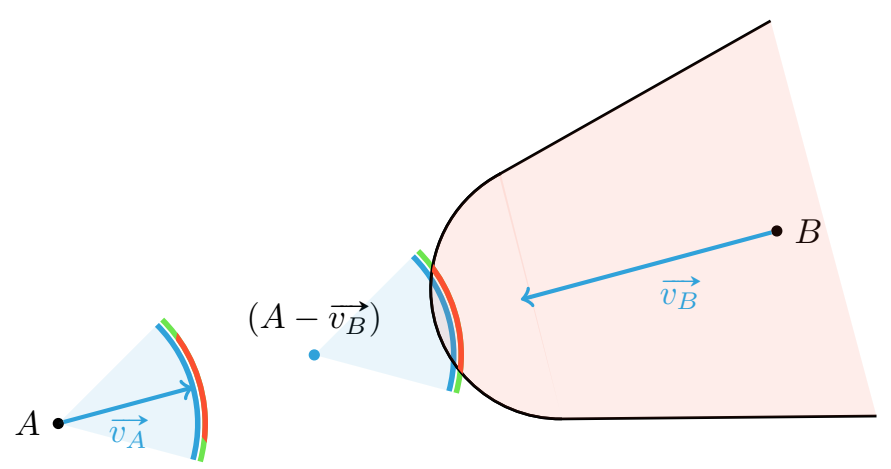

Fig. 4. Disjoint angle ranges (in green) for facing aircraft: the conflict can be solved either by turning left or right.

As it is expected that the relative performances of airliners and UASs will incur many unsolvable scenarios, for which losses of separation will occur, it may be wiser to choose an angle that leaves some leeway for the next resolution steps, i.e. which does not saturate the separation constraint.

2) Safest: To maximize the expectation of escaping a future conflict in case of further maneuvering of aircraft B (without any knowledge of its intended trajectory), the new heading could instead be chosen as the median value of the largest allowed angle range (in case of several disjoint angle ranges), which will optimize the robustness of the maneuver.

But the largest angle range must be selected before filtering the conflict-free angle ranges with the allowed turn angle (of $\pm \theta$, cf. section II-B, because these two kinds of constraints does not have the same significance: the new heading should be as far as possible from aircraft constraints (therefore chosen as the median value of the largest range) but can be arbitrarily close to the allowed turn angle constraint (e.g. the best maneuver may be to turn at the maximal possible angle). Once the corresponding angle has been computed with aircraft constraints only, the allowed turn angle constraint can be applied: if the median angle is no longer available, the closest allowed heading is chosen instead. This strategy will be referred to as Safest in the following and its result is shown in figure 5

However, the overestimate of the conflicting situation inherent to the Safest strategy can lead to unacceptable amounts of heading changes and totally disregards the UAS mission and flight plan.

3) Hybrid: Actually, the two strategies can be combined to benefit from their advantages while getting rid of their drawbacks: the Closest strategy could be favored while there is still time and space to solve potential future conflicts, resorting to the Safest one before the situation becomes dire. So we define the Hybrid strategy parameterized by $\gamma \in \mathbb{R}_{>0}$ for a set of (adverse) aircraft positions $\mathcal{F}$ at a given time step:

$$
\text { Hybrid }= \begin{cases}\text { Closest } & \text { if } \min _{\mathrm{B} \in \mathcal{F}}\|\overrightarrow{A B}\|>\gamma d \\ \text { Safest } & \text { otherwise }\end{cases}
$$

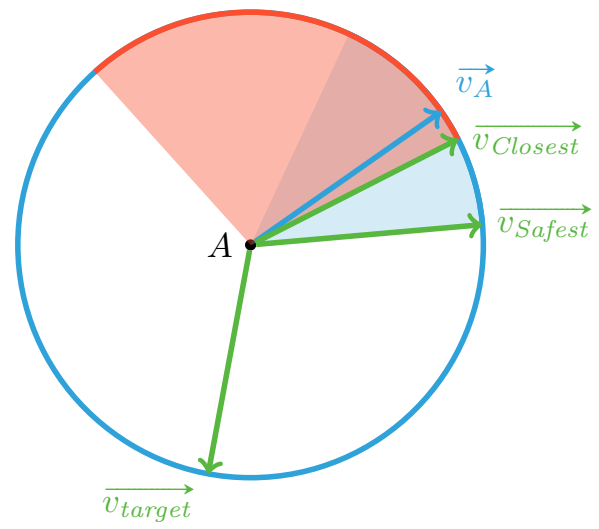

Fig. 5. Closest and Safest speeds, computed from $\overrightarrow{v_{A}}$, given the constraints in red and turn angle range in blue.

So the Closest strategy is chosen if the minimal distance between the UAS and all other aircraft is greater than $\gamma d$, and the Safest one otherwise.

Therefore, this parameter allows to continuously slide from a pure Closest strategy for $\gamma=0$ to a pure Safest strategy for $\gamma=+\infty$. The influence of parameter $\gamma$, which should a priori be chosen greater than 1 to switch to the Safest strategy when there is still enough leeway to escape the conflict, is discussed in section III-B

4) Fallback Strategy: If the permitted heading range is empty, it means that no turning angle can guarantee the separation distance $d$ for the next $\tau$ minutes. However, even if a conflict occurs, a brief one at a distance just below the target separation distance is preferable to a lasting close encounter.

To take into account this criterion while choosing a maneuver whenever a loss of separation is inevitable, our algorithm resorts to a fallback strategy consisting in reducing the separation distance until a solution can be found anew. Our current implementation uses a linear scheme with a constant step ( $0.1 \mathrm{NM}$ in our experiments) and returns the corresponding maneuver as soon as a solution is found though a dichotomous scheme could be used to speed up the search if more precision is deemed relevant.

The resulting maneuver therefore attempts to minimize the conflict, i.e. to maximize the minimal distance between the aircraft and the UAS, hopefully achieving a minimization of the conflict duration as well.

\section{Multiple Aircraft}

In real traffic situations, conflicts involving more than two aircraft often occur and cannot be solved pairwise in sequence, but must be globally handled. Fortunately, our geometrical model can be easily extended to take into account any number of aircraft.

For each aircraft, a forbidden angle range is determined exactly as in the pairwise case detailed in section II-B and removed from the possible turning range of the UAS. The resulting allowed headings may then comprise several (possibly more than two) disjoint angle ranges, because each new 


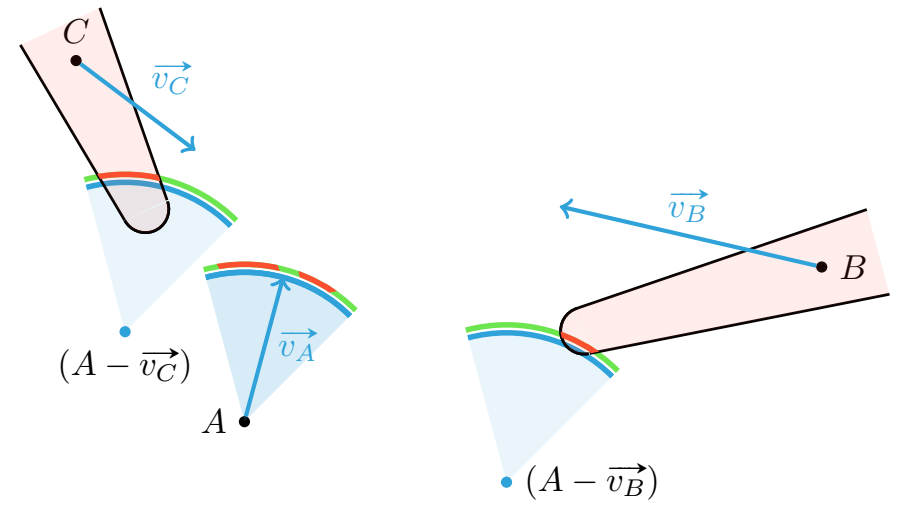

Fig. 6. Several disjoint allowed heading ranges (in green) for UAS $A$ in presence of multiple aircraft $(B$ and $C$ ).

constraint may remove a new hole in the remaining angle ranges (as illustrated in figure 6.

\section{EXPERIMENTS}

Our geometrical model has been implemented and thoroughly tested for the single aircraft case on recorded real TCA traffic enhanced by injecting various conflicting UASs scenarios. The following section described the data and scenarios generation, and the next one reports the results and analysis of our experiments.

\section{A. Test Description}

Experiments were conducted on real traffic data, recorded on 2013/09/14 in the south-west of the French airspace. This gives a much more realistic picture of the types of trajectories that UASs may encounter when flying in real traffic. This is all the more necessary because UASs are essentially meant to fly in the lower airspace where most of the commercial traffic is climbing or descending.

UASs trajectories are built from recorded tracks of aircraft with similar performances for two UAS types (flying at $80 \mathrm{kn}$ and $160 \mathrm{kn}$ respectively), three vertical profiles (leveled flight, climb and descent) and two horizontal profiles (constant heading and circle around a fixed point), for a total of 6 different patterns for each UAS type.

We filtered trajectories that had at least six minutes of flight under FL195, leaving us with a set of 475 aircraft for a total of 2850 simulation scenarios. For each aircraft and each UAS pattern defining a scenario, we then isolate a plot $\left(x_{\mathrm{AC}}, y_{\mathrm{AC}}, \mathrm{FL}_{\mathrm{AC}}\right)$ (respectively $\left.\left(x_{\mathrm{UAS}}, y_{\mathrm{UAS}}, \mathrm{FL}_{\mathrm{UAS}}\right)\right)$ in the trajectory of the aircraft (respectively the UAS) that has at least 3 minutes of past positions. These points are used to create a conflict for this scenario, i.e. we adjust the UAS trajectory in space and time dimensions so that $x_{\mathrm{AC}}=x_{\mathrm{UAS}}, y_{\mathrm{AC}}=y_{\mathrm{UAS}}$ and $\mathrm{FL}_{\mathrm{AC}}=\mathrm{FL}_{\mathrm{UAS}}$. If no maneuver were issued, there would be a collision between the aircraft and the UAS.

A fast-time simulator enables to play the trajectories (both recorded and built) and to modify them by sending maneuver messages consisting of a heading change and a turn rate.

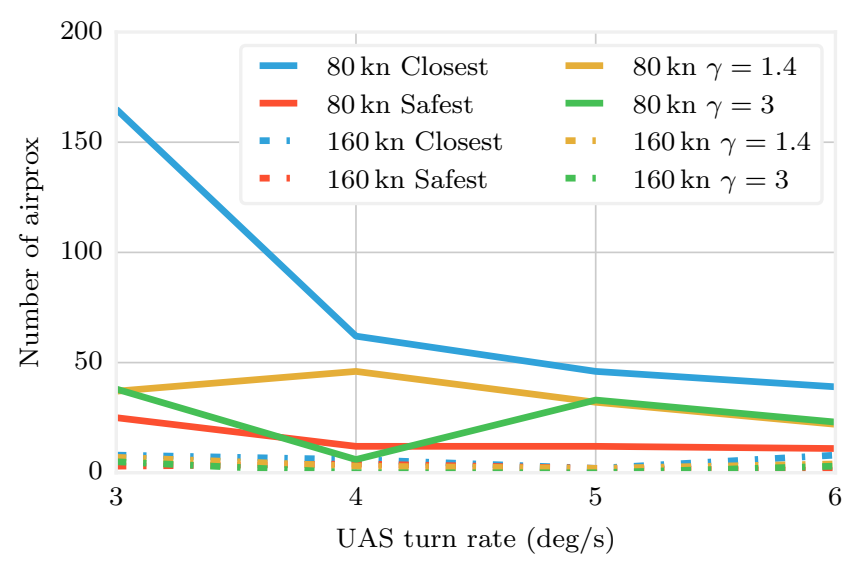

Fig. 7. Number of airprox events w.r.t. UAS turn rate. The graphs where $\gamma$ is defined correspond to the Hybrid strategy.

Those messages are send to UASs only, other aircraft are left unchanged.

In the experiments, we chose $\tau=5$ minutes and tried to achieve $3 \mathrm{NM}$ of separation when possible. We do not take the vertical separation into account because we want to test the efficiency of the detect and avoid process in the horizontal plane only. Further research will be conducted in the vertical dimension. We chose to detect and avoid conflicts every step seconds ( step $=10 \mathrm{~s}$ when not specified otherwise).

\section{B. Results with Various Speed and Turn Rate Ranges}

Fast-time simulations were run for all the 2850 scenarios on a $3.4 \mathrm{GHz}$ Intel ${ }^{\circledR}$ Xeon ${ }^{\circledR}$ workstation, each run being executed within one tenth of a second. We measured various indicators of the efficiency of the conflict avoidance maneuvers and counted the occurrences of "close distance events", which we set up to be the simulations were the distance between the UAS and the aircraft went under $1 \mathrm{NM}$. In the following, we will call an airprox (aircraft proximity) this kind of event.

Figure 7 depicts the number of airprox events as a function of the turn rate for various combinations of speed and resolution method. The speed of the UAS is clearly decisive to avoid conflicts efficiently, as the $160 \mathrm{kn}$ UAS experiences less than 10 airproxes whichever the turn rate and the strategy, whereas for slow UASs, escaping conflicts becomes easier with a greater turn rate. For the latter, the strategy is also influential and, as expected, the Safest and Hybrid ones provide better separation than the Closest strategy.

The histograms on figure 8 show the distribution of scenarios with respect to the closest distance of approach during the simulation for both Closest and Safest strategies, with a UAS flying at $160 \mathrm{kn}$ and maneuvering at $3 \% \mathrm{~s}$. We observe a peak at $3 \mathrm{NM}$ (i.e. the target separation distance) for the Closest strategy, which attests the correctness of the algorithm. Some conflicts noticeably remain in the left part of the curve, which the Safest strategy (red curve) helps to solve, although it yields larger separation distances, so that the UAS loses more time and fuel during maneuvers. 


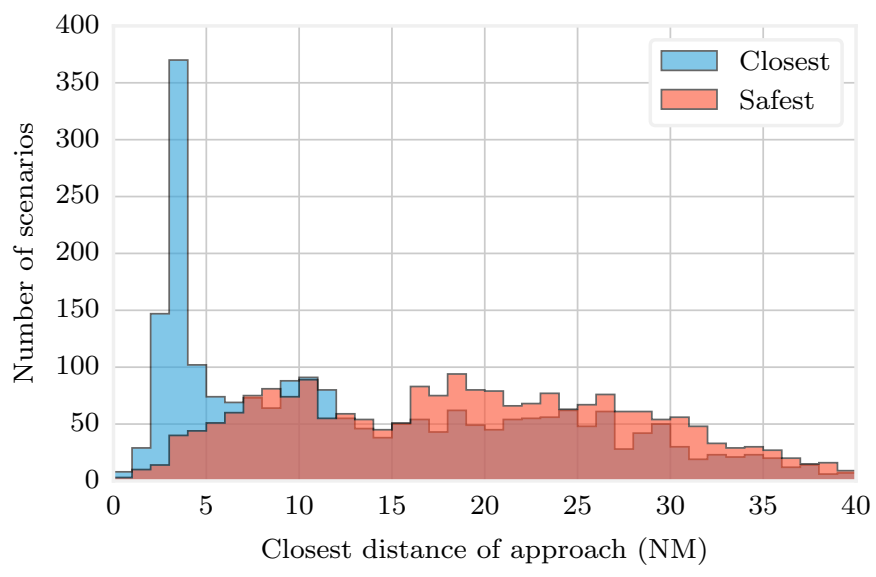

Fig. 8. Comparison of Closest (blue) and Safest (red) strategies: distribution of scenarios w.r.t. the closest distance of approach.

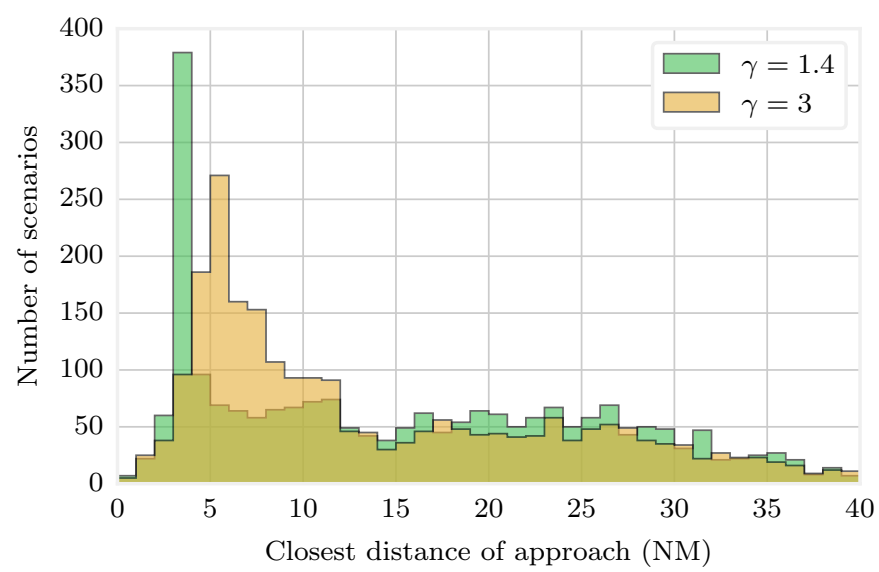

Fig. 9. Influence of parameter $\gamma$ with the Hybrid strategy: distribution of scenarios w.r.t. the closest distance of approach.

The same simulations were carried out with the Hybrid strategy for two values of parameter $\gamma$, as depicted on figure 9 exhibiting the expected trade-off between maintaining separation and limiting deviations from the original trajectories. With $\gamma=1.4$, the results are similar to the Closest strategy, however with significantly fewer losses of separation less than $3 \mathrm{NM}$. When $\gamma$ increases, the $3 \mathrm{NM}$ peak is shifted and spread towards the right part of the graph, indicating less airproxes but more costly maneuvers.

The number of airprox events is the primary indicator for the efficiency of the detect and avoid strategy. Yet, the deviation of the UAS from its trajectory and the number of maneuvers matter as well: they can be seen as the "cost" of maintaining separation or as a secondary objective to discriminate solutions with equivalent amounts of airproxes. Figure 10 shows the mean maneuver quantity, defined as the sum of the absolute values of all heading changes given to the UAS, for our three strategies (with two values of $\gamma$ for the Hybrid one) as a function of the turn rate. As expected, the

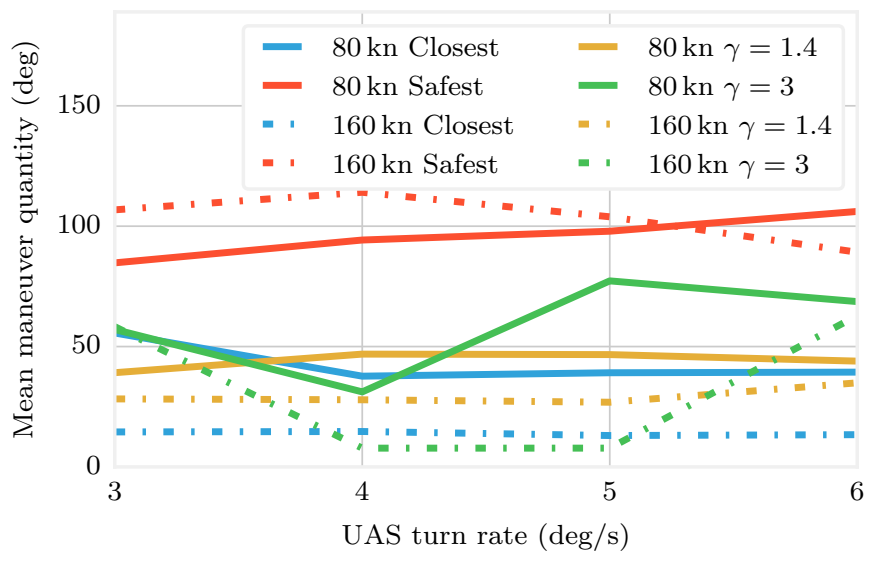

Fig. 10. Maneuver quantity w.r.t. UAS turn rate.

Safest strategy (in red) incurs the greatest maneuver quantity, whereas the Closest strategy is much more sparing. Once again, the Hybrid strategy occupies the middle ground between the two others, occasionnally providing better results than the Closest one. Not so intuitively, the turn rate seems to have little (predictable) effect on the maneuver quantity (contrarily to the number of airproxes, cf. figure 7), except for the $80 \mathrm{kn}$ UAS with the Safest strategy for which the number and range of deviation increase with the ability to take steeper turns.

\section{Conclusion And Future Work}

In the context of the integration of UASs in TCAs, we have implemented a geometrical "detect and avoid" algorithm based on data collected from ADS-B-equipped surrounding aircraft. This algorithm attempts to solve air traffic conflicts between a UAS and airliners in initial climb and approach phases by changing the UAS heading only. The geometrical resolution is based upon the model described in [3], modified with additional constraints enabling the UAS to take the responsibility of the entire maneuver at constant speed.

Once the constraints induced by the aircraft have been computed, we propose two basic strategies, one of them (Closest) leading to fewer deviations from the initial trajectory but with significant amounts of remaining airproxes, the other one (Safest) providing more robust trajectories at the cost of many more maneuvers. To overcome the drawbacks of these strategies while retaining their advantages we present a tradeoff strategy (Hybrid), switching from one to the other whenever the distance between the UAS and one of the surrounding aircraft falls below a given threshold. These methods were intensively tested on 2850 realistic traffic scenarios issued from data recorded in a French TCA.

In most cases, the aircraft was avoided as planned, with a separation greater than the target. Nevertheless, in particularly difficult circumstances where the aircraft turns out to change its heading towards the escape trajectory computed during the previous time steps, airprox events could not be avoided, especially with the Closest strategy. However, the Safest 
strategy was found very efficient at limiting the number of these occurrences, but at a high cost in terms of "maneuver quantity". Eventually, the Hybrid strategy was found the most promising one, with a threshold ranging from one to three separation norms. Further experiments need to be performed to identify the Pareto front w.r.t. this threshold parameter.

One of the pitfalls of our method is that it only takes into account the current state, so that any further change in the aircraft state could break the resolution, especially with the Closest strategy. In order to improve the robustness of the maneuvers, we plan to try and anticipate better, both on the aircraft intentions and the UAS capabilities.

Knowing the past positions of the aircraft, it is possible to build a short-term predicted trajectory, based on the analysis of the derivatives of its speed and turn angle. For example, the beginning or the end of a turn, a climb or a descent could be inferred. Particular care would have to be taken during the calibration phase, especially when choosing the number of past states to consider: too much states would create some latency in the prediction, whereas too few states would yield unreliable ones.

If the aircraft trajectory could be predicted this way, then it becomes particularly interesting to anticipate several maneuvers for the UAS. This could be planned optimally with an $\mathrm{A}^{*}$ or Dijkstra algorithm, using the geometrical algorithm at each step to prune the search tree or validate the existence of partial solutions, at the cost of a significantly longer computation. It could also be performed geometrically by an approximation of a few maneuvers aggregated into a single one.

For the hardest scenarios, we plan to check the interaction of the current algorithm with the TCAS of airliners and, if necessary, to implement a vertical strategy for the UAS that could comply with airliner TCAS maneuvers.

\section{REFERENCES}

[1] ABIresearch, "Small unmanned aerial systems market exceeds US $\$ 8.4$ billion by 2019 , dominated by the commercial sector and driven by commercial applications," January 2015.

[2] DCF, Sagem, DSNA, and ENAC, "ODREA demonstration report," tech. rep., SESAR Joint Undertaking, 2015.

[3] J. van den Berg, S. J. Guy, M. C. Lin, and D. Manocha, "Reciprocal $n$-body Collision Avoidance," in 14th International Symposium on Robotics Research, pp. 3-19, 2011.

[4] N. Durand and N. Barnier, "Does ATM need centralized coordination? Autonomous conflict resolution analysis in a constrained speed environment," in 11th ATM R and D Seminar, 2015.

[5] K. Zeghal, "A comparison of different approaches based on force fields for coordination among multiple mobiles," in Intelligent Robots and Systems, 1998. Proceedings., 1998 IEEE/RSJ International Conference on, vol. 1, pp. 273-278 vol.1, Oct 1998 .
[6] J. Kosecka, C. Tomlin, G. Pappas, and S. Sastry, "2-1/2 D Conflict Resolution Maneuvers for ATMS," in in Proc. 37th IEEE Conf. Decision Control, pp. 2650-2655, 1998.

[7] M. Eby and I. Kelly, W.E., "Free flight separation assurance using distributed algorithms," in Aerospace Conference, 1999. Proceedings. 1999 IEEE, vol. 2, pp. 429-441 vol.2, 1999.

[8] G. Granger, N. Durand, and J. Alliot, "Token allocation strategy for free-flight conflict solving," in IJCAI'01, 2001.

[9] G. Granger, N. Durand, and J. Alliot, "Optimal resolution of en route conflicts," in 4th ATM R and D Seminar, 2001.

[10] N. Archambault and N. Durand, "Scheduling heuristics for on-board sequential air conflict solving," in Digital Avionics Systems Conference, 2004. DASC 04. The 23rd, vol. 1, pp. -3.1-9 Vol.1, Oct 2004.

[11] N. Durand, J. Alliot, and F. Medioni, "Neural nets trained by genetic algorithms for collision avoidance," Applied Artificial Intelligence, 2000.

[12] I. Hwang, J.Kim, and C. Tomlin, "Protocol-based conflict resolution for air traffic control," ATC Quarterly, vol. 15, pp. 1-34, 2007.

[13] J. Le Ny and G. Pappas, "Geometric programming and mechanism design for air traffic conflict resolution," in American Control Conference (ACC), 2010, pp. 3069-3074, June 2010.

[14] J. Snape and D. Manocha, "Navigating multiple simple-airplanes in 3D workspace," in IEEE International Conference on Robotics and Automation (ICRA), pp. 3974-3980, 2010.

[15] N. Durand, J.-M. Alliot, and J. Noailles, "Automatic aircraft conflict resolution using genetic algorithms," in Proceedings of the Symposium on Applied Computing, Philadelphia, ACM, 1996.

[16] F. Krella et al., "Arc 2000 scenario (version 4.3)," tech. rep., Eurocontrol, April 1989.

[17] Y.-J. Chiang, J. Klosowski, C. Lee, and J. Mitchell, "Geometric algorithms for conflict detection/resolution in air traffic management," in Decision and Control, 1997., Proceedings of the 36th IEEE Conference on, vol. 2, pp. 1835-1840 vol.2, Dec 1997.

[18] J. Hu, M. Prandini, A. Nilim, and S. Sastry, "Optimal coordinated maneuvers for three dimensional aircraft conflict resolution," $A I A A$ Journal of Guidance, Control and Dynamics, vol. 25, p. 2002, 2002.

[19] J.-H. Oh, J. Shewchun, and E. Feron, "Design and analysis of conflict resolution algorithms via positive semidefinite programming [aircraft conflict resolution]," in Decision and Control, 1997., Proceedings of the 36th IEEE Conference on, vol. 5, pp. 4179-4185 vol.5, Dec 1997.

[20] E. Frazzoli, Z.-H. Mao, J.-H. Oh, and E. Feron, "Resolution of conflicts involving many aircraft via semidefinite programming," AIAA Journal of Guidance, Control and Dynamics, vol. 24, Jan-Feb 2001.

[21] L. Pallottino, A. Bicchi, and E. Feron, "Mixed integer programming for aircraft conflict resolution," in AIAA Guidance Navigation and Control Conference and Exhibit, 2001.

[22] L. Pallottino, E. Feron, and A. Bicchi, "Conflict resolution problems for air traffic management systems solved with mixed integer programming," Intelligent Transportation Systems, IEEE Transactions on, vol. 3, pp. 3-11, Mar 2002.

[23] M. A. Christodoulou and C. Kontogeorgou, "Collision avoidance in commercial aircraft free flight via neural networks and non-linear programming," Int. J. Neural Syst., vol. 18, no. 5, pp. 371-387, 2008.

[24] M. Gariel and E. Feron, "3D conflict avoidance under uncertainties," in Digital Avionics Systems Conference, 2009. DASC '09. IEEE/AIAA 28th, pp. 4.E.3-1-4.E.3-8, Oct 2009.

[25] N. Durand and J.-M. Alliot, "Optimal resolution of en route conflicts," in First ATM Seminar Europe/USA, Saclay, 1997.

[26] C. Allignol, N. Barnier, N. Durand, and J.-M. Alliot, "A New Framework for Solving En-Route Conflicts," in 10th USA/Europe Air Traffic Management Research and Developpment Seminar, 2013. 